\title{
MULTIPLICATIVE PROPERTIES OF POSITIVE MAPS
}

\author{
ERLING STØRMER
}

(Dedicated to the memory of Gert K. Pedersen)

\begin{abstract}
Let $\phi$ be a positive unital normal map of a von Neumann algebra $M$ into itself. It is shown that with some faithfulness assumptions on $\phi$ there exists a largest Jordan subalgebra $C_{\phi}$ of $M$ such that the restriction of $\phi$ to $C_{\phi}$ is a Jordan automorphism and each weak limit point of $\left(\phi^{n}(a)\right)$ for $a \in M$ belongs to $C_{\phi}$.
\end{abstract}

\section{Introduction}

In the study of positive linear maps of $C^{*}$-algebras the multiplicative properties of such maps have been studied by several authors, see e.g. [9], [2], [3], [4], [6]. If $\phi: A \rightarrow B$ is a positive unital map between $C^{*}$-algebras $A$ and $B$ an application of Kadison's Schwarz inequality, [8] to the operators $a+a^{*}$ and $i\left(a-a^{*}\right)$ yields the inequality [10]

$$
\phi\left(a \circ a^{*}\right) \geq \phi(a) \circ \phi(a)^{*}, \quad a \in A,
$$

where $a \circ b=\frac{1}{2}(a b+b a)$ is the Jordan product. Thus one obtains an operator valued sesquilinear form

$$
\langle a, b\rangle=\phi\left(a \circ b^{*}\right)-\phi(a) \circ \phi(b)^{*}, \quad a, b \in A .
$$

If we apply the Cauchy-Schwarz inequality to $\omega(\langle a, b\rangle)$ for all states $\omega$ of $B$ it was noticed in [6] that if $\phi\left(a \circ a^{*}\right)=\phi(a) \circ \phi(a)^{*}$ then $\langle a, b\rangle=0$ for all $b \in A$. We call the set

$$
A_{\phi}=\left\{a \in A: \phi\left(a \circ a^{*}\right)=\phi(a) \circ \phi(a)^{*}\right\}
$$

the definite set of $\phi$. It is a Jordan subalgebra of $A$, and if $a \in A_{\phi}$ then $\phi(a \circ b)=\phi(a) \circ \phi(b)$ for all $b \in A$.

In the present paper we shall develop the theory further. We first study positive unital normal, i.e. ultra weakly continuous, maps $\phi: M \rightarrow M$, where

Received June 22, 2006. 
$M$ is a von Neumann algebra. We mainly study properties of the definite set $M_{\phi}$ and some of its Jordan subalgebras of $M$ plus convergence properties of the orbits $\left(\phi^{n}(a)\right)$ for $a \in M$. We shall show that when there exists a faithful family $\mathscr{F}$ of $\phi$-invariant normal states there is a largest Jordan subalgebra $C_{\phi}$ of $M$ called the multiplicative core of $M$, on which $\phi$ acts as a Jordan automorphism. Furthermore if $a \in M$ then every weak limit point of the orbit $\left(\phi^{n}(a)\right)$ lies in $C_{\phi}$, and if $\rho(a \circ b)=0$ for all $b \in C_{\phi}$, then $\phi^{n}(a) \rightarrow 0$ weakly.

Much of the above work was inspired by a theorem of Arveson, [1]. In the last section we study the $C^{*}$-algebra case and the relation of our discussion with Arveson's work. Then $\phi: A \rightarrow A$ is a positive unital map, and we assume the orbits $\left(\phi^{n}(a)\right)$ with $a \in A$ are norm relatively compact and that there exists a faithful family $\mathscr{F}$ of $\phi$-invariant states. It is then shown that the multiplicative core $C_{\phi}$ of $\phi$ equals the set of main interest in [1], namely the norm closure of the linear span of all eigenoperators $a \in A$ with $\phi(a)=\lambda a,|\lambda|=1$, and that $\lim _{n \rightarrow \infty}\left\|\phi^{n}(a)\right\|=0$ if and only if $\rho(a \circ b)=0$ for all $b \in C_{\phi}$ and $\rho \in \mathscr{F}$.

\section{Maps on von Neumann algebras}

Throughout this section $M$ denotes a von Neumann algebra, $\phi: M \rightarrow M$ is a positive normal unital map. $M_{\phi}$ denotes the definite set of $\phi$ and $\langle$,$\rangle the operator$ valued sesquilinear form $\langle a, b\rangle=\phi\left(a \circ b^{*}\right)-\phi(a) \circ \phi(b)^{*}, a, b \in A$.

Lemma 2.1. Let assumptions be as above, and suppose $\left(a_{\alpha}\right)$ is a bounded net in $M$ which converges weakly to $a \in M$. If $\left\langle a_{\alpha}, a_{\alpha}\right\rangle \rightarrow 0$ weakly, then $a \in M_{\phi}$, and $\phi(a \circ b)=\phi(a) \circ \phi(b)$ for all $b \in M$.

Proof. Let $\omega$ be a normal state on $M$. By the Cauchy-Schwarz inequality, if $b, c \in M$ we have

$$
|\omega(\langle b, c\rangle)|^{2} \leq \omega(\langle b, b\rangle) \omega(\langle c, c\rangle) .
$$

By assumption, if $a_{\alpha}$ and $a$ are as in the statement of the lemma, and $b \in M$ then

$$
\begin{aligned}
|\omega(\langle a, b\rangle)|^{2} & =\lim _{\alpha}\left|\omega\left(\left\langle a_{\alpha}, b\right\rangle\right)\right|^{2} \\
& \leq \lim _{\alpha} \omega\left(\left\langle a_{\alpha}, a_{\alpha}\right\rangle\right) \omega(\langle b, b\rangle)=0 .
\end{aligned}
$$

Since this holds for all normal states $\omega,\langle a, b\rangle=0$, completing the proof.

In analogy with the definition of G-finite for automorphism groups we introduce

Definition 2.2. With $\phi$ as above we say $M$ is $\phi$-finite if there exists a faithful family $\mathscr{F}$ of $\phi$-invariant normal states on the von Neumann algebra generated by the image $\phi(M)$. 
Lemma 2.3. Assume $M$ is $\phi$-finite. Then for $a \in M$ we have

(i) Every weak limit point of the orbit $\left(\phi^{n}(a)\right)$ of a belongs to $M_{\phi}$.

(ii) If $\rho\left(\phi^{n}(a) \circ b\right)=0$ for all $b \in M_{\phi}, \rho \in \mathscr{F}$, then $\phi^{n}(a) \rightarrow 0$ weakly.

Proof. If $\rho \in \mathscr{F}$ denote by $\|\cdot\|_{\rho}$ the seminorm $\|x\|_{\rho}=\rho\left(x \circ x^{*}\right)^{\frac{1}{2}}$. Then by the inequality (1)

$$
\begin{aligned}
\left\|\phi^{n+1}(a)\right\|_{\rho}^{2} & =\rho\left(\phi^{n+1}(a) \circ \phi^{n+1}(a)^{*}\right) \\
& \leq \rho\left(\phi\left(\phi^{n}(a) \circ \phi^{n}(a)^{*}\right)\right) \\
& =\left\|\phi^{n}(a)\right\|_{\rho}^{2} .
\end{aligned}
$$

Thus the sequence $\left\|\phi^{n}(a)\right\|_{\rho}^{2}$ is decreasing, hence $\left\|\phi^{n}(a)\right\|_{\rho}^{2}-\left\|\phi^{n+1}(a)\right\|_{\rho}^{2} \rightarrow$ 0 . We have

$$
\begin{aligned}
\rho\left(\left\langle\phi^{n}(a), \phi^{n}(a)\right\rangle\right) & =\rho\left(\phi\left(\phi^{n}(a) \circ \phi^{n}(a)^{*}\right)-\phi\left(\phi^{n}(a)\right) \circ \phi\left(\phi^{n}(a)^{*}\right)\right) \\
& =\rho\left(\phi^{n}(a) \circ \phi^{n}(a)^{*}-\phi^{n+1}(a) \circ \phi^{n+1}(a)^{*}\right) \\
& =\left\|\phi^{n}(a)\right\|_{\rho}^{2}-\left\|\phi^{n+1}(a)\right\|_{\rho}^{2} \rightarrow 0 .
\end{aligned}
$$

Since this hold for all $\rho \in \mathscr{F}$ and $\mathscr{F}$ is faithful, $\left\langle\phi^{n}(a), \phi^{n}(a)\right\rangle \rightarrow 0$ weakly. By Lemma 2.1, if $a_{0}$ is a weak limit point of $\left(\phi^{n}(a)\right)$ then $a_{0} \in M_{\phi}$, proving (i).

To show (ii) suppose $\rho\left(\phi^{n}(a) \circ b\right)=0$ for all $b \in M_{\phi}, \rho \in \mathscr{F}$. Let $a_{0}$ be a weak limit point of $\left(\phi^{n}(a)\right)$. Then $\rho\left(a_{0} \circ b\right)=0$ for all $b \in M_{\phi}$, in particular by part (i) $\rho\left(a_{0} \circ a_{0}\right)=0$. Since $\mathscr{F}$ is faithful on the von Neumann algebra generated by $\phi(M), a_{0}=0$. Thus 0 is the only weak limit point of $\left(\phi^{n}(a)\right)$, so $\phi^{n}(a) \rightarrow 0$ weakly. The proof is complete.

It is not true in general that $\phi\left(M_{\phi}\right) \subseteq M_{\phi}$. We therefore introduce the following auxiliary concept. If $\phi: A \rightarrow A$ is positive unital with $A$ a $C^{*}$ algebra, then $A_{\Phi}=\left\{a \in A_{\phi}: \phi^{k}(a) \in A_{\phi}, k \in \mathrm{N}\right\}$.

Lemma 2.4. Let $M$ be $\phi$-finite and $M_{\Phi}$ defined as above. Then $M_{\Phi}$ is a weakly closed Jordan subalgebra of $M_{\phi}$ such that $\phi\left(M_{\Phi}\right) \subseteq M_{\Phi}$, and if $a \in M$ then every weak limit point of $\left(\phi^{n}(a)\right)$ belongs to $M_{\Phi}$. Furthermore, if $\rho\left(\phi^{n}(a) \circ b\right)=0$ for all $b \in M_{\Phi}, \rho \in \mathscr{F}$, then $\phi^{n}(a) \rightarrow 0$ weakly.

Proof. Since $M$ is weakly closed and $\phi$ is weakly continuous on bounded sets $M_{\Phi}$ is weakly closed. Since $\phi$ and its powers $\phi^{k}$ are Jordan homomorphisms on $M_{\Phi}$ it is straightforward to show $M_{\Phi}$ is a Jordan subalgebra of $M$. Furthermore it is clear from its definition that $\phi\left(M_{\Phi}\right) \subseteq M_{\Phi}$.

If $a \in M$ and $a_{0}$ is a weak limit point of $\left(\phi^{n}(a)\right)$, then $a_{0} \in M_{\phi}$ by Lemma 2.3. Then $\phi\left(a_{0}\right)$ is a weak limit point of $\left(\phi^{n+1}(a)\right)$, hence belongs to $M_{\phi}$, 
again by Lemma 2.3. Iterating we have $\phi^{k}\left(a_{0}\right) \in M_{\phi}$ for all $k \in \mathrm{N}$. Thus $a_{0} \in M_{\Phi}$. The last statement follows exactly as in Lemma 2.3. The proof is complete.

It is not true that $\phi\left(M_{\Phi}\right)=M_{\Phi}$. To remedy this problem we introduce yet another Jordan subalgebra.

Definition 2.5. Let $\phi: A \rightarrow A$ be positive unital with $A$ a $C^{*}$-algebra. The multiplicative core of $\phi$ is the set

$$
C_{\phi}=\bigcap_{n=0}^{\infty} \phi^{n}\left(A_{\Phi}\right) .
$$

Lemma 2.6. $C_{\phi}$ satisfies the following:

(i) $C_{\phi}$ is a Jordan subalgebra of $A$.

(ii) $\phi\left(C_{\phi}\right)=C_{\phi}$.

Suppose the restriction of $\phi$ to $C_{\phi}$ is faithful. Then we have

(iii) The restriction of $\phi$ to $C_{\phi}$ is a Jordan automorphism.

(iv) $C_{\phi}$ is the largest Jordan subalgebra of $A$ on which the restriction of $\phi$ is a Jordan automorphism.

Proof. As in Lemma $2.4 C_{\phi}$ is clearly a Jordan subalgebra of $A$ such that $\phi\left(C_{\phi}\right) \subseteq C_{\phi}$ and is weakly closed in the von Neumann algebra case. Furthermore, since $\phi\left(A_{\Phi}\right) \subseteq A_{\Phi}$, we have $\phi^{n}\left(A_{\Phi}\right) \subseteq \phi^{n-1}\left(A_{\Phi}\right)$, so that the sequence $\left(\phi^{n}\left(A_{\Phi}\right)\right)$ is decreasing. Thus

$$
C_{\phi}=\bigcap_{n=0}^{\infty} \phi^{n+1}\left(A_{\Phi}\right)=\phi\left(C_{\phi}\right),
$$

so (i) and (ii) are proved.

We next show (iii). By (ii) the restriction of $\phi$ to $C_{\phi}$ is a Jordan homomorphism of $C_{\phi}$ onto itself. In particular since $\phi$ is faithful on $C_{\phi}$, it is a Jordan automorphism of $C_{\phi}$, proving (iii).

To show (iv) let $B$ be a Jordan subalgebra of $A$ such that $\left.\phi\right|_{B}$ is a Jordan automorphism of $B$. Then clearly $B \subseteq A_{\Phi}$, and $\phi^{n}(B)=B$, so that

$$
B=\bigcap_{n=0}^{\infty} \phi^{n}(B) \subseteq \bigcap_{n=0}^{\infty} \phi^{n}\left(A_{\Phi}\right)=C_{\phi} .
$$

The proof is complete.

We can now prove our main result. 
THEOREM 2.7. Let $M$ be $\phi$-finite, and $\mathscr{F}$ a set of normal $\phi$-invariant states which is faithful on the von Neumann algebra generated by $\phi(M)$. Let $a \in M$. Then we have

(i) Every weak limit point of $\left(\phi^{n}(a)\right)$ lies in $C_{\phi}$.

(ii) If $\rho(a \circ b)=0$ for all $b \in C_{\phi}, \rho \in \mathscr{F}$, then $\phi^{n}(a) \rightarrow 0$ weakly.

Proof. (i) Let $a_{0}$ be a weak limit point of $\left(\phi^{n}(a)\right)$. By Lemma $2.4 a_{0} \in M_{\Phi}$. Choose a subnet $\left(\phi^{n_{\alpha}}(a)\right)$ which converges weakly to $a_{0}$. Let $k \in \mathrm{N}$, and let $\left(\phi^{m_{\beta}}(a)\right)$ be a subnet of $\left(\phi^{n_{\alpha}-k}(a)\right)$ which converges weakly to $a_{1} \in M_{\Phi}$ (again using Lemma 2.4, since $\left(\phi^{m_{\beta}}(a)\right)$ will be a subnet of $\left.\left(\phi^{n}(a)\right)\right)$. Each $m_{\beta}$ is of the form $n_{\alpha_{j}}-k$. The net $\left(\phi^{n_{\alpha_{j}}}(a)\right)$ converges to $a_{0}$, since it is a subnet of the converging net $\left(\phi^{n_{\alpha}}(a)\right)$. Thus we have

$$
\begin{aligned}
\phi^{k}\left(a_{1}\right) & =\lim \phi^{k}\left(\phi^{m_{\beta}}(a)\right) \\
& =\lim \phi^{k+\left(n_{\alpha_{j}}-k\right)}(a) \\
& =\lim \phi^{n_{\alpha_{j}}}(a) \\
& =a_{0} .
\end{aligned}
$$

Thus $a_{0} \in \phi^{k}\left(M_{\Phi}\right)$ for all $k \in \mathrm{N}$, hence $a_{0} \in C_{\phi}$.

To show (ii) suppose $\rho(a \circ b)=0$ for all $\rho \in \mathscr{F}, b \in \mathrm{C}_{\phi}$. Since $\phi^{k}\left(C_{\phi}\right)=$ $C_{\phi}$ there exists $c \in C_{\phi}$ such that $b=\phi^{k}(c)$. Thus

$$
\begin{aligned}
\rho\left(\phi^{k}(a) \circ b\right) & =\rho\left(\phi^{k}(a) \circ \phi^{k}(c)\right) \\
& =\rho\left(\phi^{k}(a \circ c)\right) \\
& =\rho(a \circ c)=0 .
\end{aligned}
$$

By part (i) every weak limit point $a_{0}$ of ( $\left.\phi^{n}(a)\right)$ lies in $C_{\phi}$, so it follows by the above that $\rho\left(a_{0} \circ b\right)=0$ for all $b \in C_{\phi}$. In particular $\rho\left(a_{0} \circ a_{0}\right)=0$, so by faithfulness of $\mathscr{F}, a_{0}=0$, hence $\phi^{n}(a) \rightarrow 0$ weakly. The proof is complete.

One might believe that the converse of part (ii) in the above theorem is true. This is false. Indeed, let $M_{0}$ be a von Neumann algebra with a faithful normal tracial state $\tau_{0}$. Let $M_{i}=M_{0}, \tau_{i}=\tau_{0}, i \in \mathrm{Z}$, and let $M=\bigotimes_{-\infty}^{\infty}\left(M_{i}, \tau_{i}\right)$. Let $\phi$ be the shift to the right. Then $C_{\phi}=M$. However, if $a=\ldots 1 \otimes a_{0} \otimes 1 \ldots \in M$ with $a_{0} \in M_{0}$, then $\lim _{n \rightarrow \infty} \phi^{n}(a)=\tau_{0}\left(a_{0}\right) 1$, so if $\tau_{0}\left(a_{0}\right)=0$, then the weak limit is 0 . But $\tau(a \circ b) \neq 0$ for some $b \in M=C_{\phi}$.

If we assume convergence in the strong-* topology then the converse holds, as we have

Proposition 2.8. Let $M$ be $\phi$-finite. Let $a \in M$ and suppose the sequence $\left(\phi^{n}(a)\right)$ converges in the strong-* topology. Then $\rho(a \circ b)=0$ for all $b \in$ $C_{\phi}, \rho \in \mathscr{F}$ if and only if $\phi^{n}(a) \rightarrow 0 *_{-}$strongly. 
Proof. If $\rho(a \circ b)=0$ for all $b \in C_{\phi}, \rho \in \mathscr{F}$ then $\phi^{n}(a) \rightarrow 0$ weakly by the theorem. Since the sequence converges *-strongly the limit must be 0 .

Conversely, if $\phi^{n}(a) \rightarrow 0 *$-strongly, then for all $b \in C_{\phi}, \rho \in \mathscr{F}$

$$
\rho(a \circ b)=\rho\left(\phi^{n}(a \circ b)\right)=\rho\left(\phi^{n}(a) \circ \phi^{n}(b)\right) \rightarrow 0,
$$

since multiplication is *-strongly continuous on bounded sets. The proof is complete.

We have not in general found a nice description of the complement of $C_{\phi}$ in $M$, i.e. a subspace $D$ such that $M$ is a direct sum of $C_{\phi}$ and $D$. In the finite case with a faithful normal $\phi$-invariant trace this can be done.

Proposition 2.9. Suppose $M$ has a faithful normal $\phi$-invariant tracial state. Then there exists a faithful normal positive projection $P: M \rightarrow C_{\phi}$ which commutes with $\phi$. Let $D=\{a-P(a): a \in M\}$. Then $M=C_{\phi}+D$ is a direct sum, and if $a \in D$ then $\phi^{n}(a) \rightarrow 0$ weakly.

Proof. Since $M$ is finite the same construction as that of trace invariant conditional expectations onto von Neumann subalgebras yields the existence of a faithful trace invariant positive normal projection $P: M \rightarrow C_{\phi}$, see [7]. Let $\tau$ be the trace alluded to in the proposition. Since $\tau$ is faithful and $\phi$-invariant, $\phi$ has an adjoint map $\phi^{*}: M \rightarrow M$ defined by $\tau\left(a \phi^{*}(b)\right)=\tau(\phi(a) b)$ for $a, b \in$ $M$. Clearly $\phi^{*}$ is $\tau$-invariant, positive, unital, and normal, and its extension $\bar{\phi}^{*}$ to an operator on $L^{2}(M, \tau)$ is the usual adjoint of the extension $\bar{\phi}$ of $\phi$. Since the restriction of $\bar{\phi}$ to the closure $C_{\phi}^{-}$of $C_{\phi}$ in $L^{2}(M, \tau)$ is an isometry of $C_{\phi}^{-}$ onto itself, so is $\bar{\phi}^{*}$. It follows that $\phi P=P \phi P=\left(P \phi^{*} P\right)^{*}=\left(\phi^{*} P\right)^{*}=P \phi$.

It is clear that $M=C_{\phi}+D$ is a direct sum. Suppose $a \in D$, i.e. $P(a)=0$. Then $\tau(a \circ b)=0$ for all $b \in C_{\phi}$. If we let $\mathscr{F}=\left\{\left.\tau\right|_{C_{\phi}} \circ P\right\}$ then, since $P$ commutes with $\phi, \mathscr{F}$ is a faithful family of normal $\phi$-invariant states. By Theorem $2.7 \phi^{n}(a) \rightarrow 0$ weakly, proving the proposition.

\section{Maps of $C^{*}$-algebras}

Arveson [1] proved the following result.

Theorem 3.1 (Arveson). Let A be a $C^{*}$-algebra, $\phi: A \rightarrow$ A a completely positive contraction such that the orbit $\left(\phi^{n}(a)\right)$ is norm relative compact for all $a \in A$. Then there exists a completely positive projection $P: A \rightarrow A$ onto the norm closed linear span $E_{\phi}$ of the eigenoperators $a \in A$ with $\phi(a)=\lambda a$, with $|\lambda|=1$, and $\alpha=\left.\phi\right|_{E_{\phi}}$ is a complete isometry of $E_{\phi}$ onto itself. We have

$$
\lim _{n \rightarrow \infty}\left\|\phi^{n}(a)-(\alpha \circ P)^{n}(a)\right\|=0,
$$


and $A$ is the direct sum of $E_{\phi}$ and the set $\left\{a \in A: \lim _{n}\left\|\phi^{n}(a)\right\|=0\right\}$.

We shall now show how our previous results yield a result which is in a sense complementary to Arveson's theorem.

Theorem 3.2. Let $A$ be a unital $C^{*}$-algebra and $\phi: A \rightarrow A$ a positive unital map such that the orbit $\left(\phi^{n}(a)\right)$ is norm relative compact for all $a \in A$. Let $C_{\phi}$ be the multiplicative core for $\phi$ in $A$, and let $E_{\phi}$ denote the set of eigenoperators $a \in A$ such that $\phi(a)=\lambda a$, with $|\lambda|=1$. Assume there exists a set $\mathscr{F}$ of $\phi$-invariant states which is faithful on the $C^{*}$-algebra generated by $\phi(A)$. Then we have

(i) $E_{\phi}=C_{\phi}$ is a Jordan subalgebra of $A$.

(ii) The restriction $\left.\phi\right|_{E_{\phi}}$ is a Jordan automorphism of $E_{\phi}$.

(iii) Let $a \in A$. Then $\rho(a \circ b)=0$ for all $\rho \in \mathscr{F}, b \in C_{\phi}$ if and only if $\lim _{n \rightarrow \infty}\left\|\phi^{n}(a)\right\|=0$.

Proof. We first show (ii). If $\phi(a)=\lambda a$ then $\phi\left(a^{*}\right)=\bar{\lambda} a^{*}$, so $E_{\phi}$ is self-adjoint. Furthermore by inequality (1)

$$
\phi\left(a \circ a^{*}\right) \geq \phi(a) \circ \phi\left(a^{*}\right)=\lambda a \circ \bar{\lambda} a^{*}=a \circ a^{*} .
$$

Composing by $\rho \in \mathscr{F}$ and using that $\mathscr{F}$ is faithful on $C^{*}(\phi(A))$ it follows that $\phi\left(a \circ a^{*}\right)=\phi(a) \circ \phi\left(a^{*}\right)$, so $a \in A_{\phi}$, the definite set of $\phi$. Since $a \in E_{\phi}$ is an eigenoperator, so is $a^{2}$, hence $E_{\phi}$ is a Jordan subalgebra of $A_{\phi}$. Note that if $\phi(a)=\lambda a$ then $\phi(\phi(a))=\phi(\lambda a)=\lambda \phi(a)$, so $\phi(a) \in E_{\phi}$. Thus $\phi: E_{\phi} \rightarrow E_{\phi}$. If $a=\sum \mu_{i} a_{i} \in E_{\phi}$ where $\phi\left(a_{i}\right)=\lambda_{i} a_{i}$, then $a=$ $\sum \mu_{i} \bar{\lambda}_{i} \phi\left(a_{i}\right) \in \phi\left(E_{\phi}\right)$, so by density of such $a^{\prime}$ s, $\phi\left(E_{\phi}\right)=E_{\phi}$. Thus by faithfulness of $\mathscr{F}$ the restriction $\left.\phi\right|_{E_{\phi}}$ is a Jordan automorphism, proving (ii).

It follows from Lemma 2.6 that $E_{\phi} \subseteq C_{\phi}$. To show the converse inclusion we use that the orbit $\left(\phi^{n}(a)\right)_{n \in \mathrm{N}}$ is norm relative compact for all $a \in A$. By Lemma 2.6 the restriction of $\phi$ to $C_{\phi}$ is a Jordan automorphism, hence in particular an isometry. We assert that if $a \in C_{\phi}$ then the orbit $\left(\phi^{n}(a)\right)_{n \in Z}$ is relative norm compact. For this it is enough to show that the set $\left(\phi^{-n}(a)\right)_{n \in \mathrm{N}}$ is relative norm compact, or equivalently that each sequence $\left(\phi^{-n_{k}}(a)\right)$ has a convergent subsequence. By assumption $\left(\phi^{n_{k}}(a)\right)$ has a convergent subsequence $\left(\phi^{m_{l}}(a)\right)$. Since this sequence is Cauchy, and

$$
\left\|\phi^{-n}(a)-\phi^{-m}(a)\right\|=\left\|\phi^{n+m}\left(\phi^{-n}(a)-\phi^{-m}(a)\right)\right\|=\left\|\phi^{n}(a)-\phi^{m}(a)\right\|,
$$

it follows that $\left(\phi^{-m_{l}}(a)\right)$ is Cauchy, and therefore converges. Thus the set $\left(\phi^{-n}(a)\right)_{n \in \mathrm{N}}$ is relative norm compact, as is $\left(\phi^{n}(a)\right)_{n \in \mathrm{Z}}$. By a well known result on almost periodic groups, see e.g. Lemma 2.8 in [1], $\left.\phi\right|_{C_{\phi}}$ has pure point spectrum. Thus $C_{\phi} \subseteq E_{\phi}$, proving (i). 
It remains to show (iii). As in the proof of Lemma 2.3 we find that every norm limit point $a_{0}$ of ( $\left.\phi^{n}(a)\right)$ belongs to $A_{\phi}$, and by the proof of Lemma 2.4 $a_{0} \in A_{\Phi}=\left\{x \in A_{\phi}: \phi^{k}(x) \in A_{\phi}, k \in \mathrm{N}\right\}$. A straightforward modification of the proof of Theorem 2.7(i), replacing weak by norm, shows that $a_{0} \in C_{\phi}$. Let $a \in A$ satisfy $\rho(a \circ b)=0$ for all $b \in C_{\phi}, \rho \in \mathscr{F}$. Then by the proof of Theorem 2.7(ii), every norm limit point of $\left(\phi^{n}(a)\right)$ is 0 . Thus there is a subsequence $\left(\phi^{n_{k}}(a)\right)$ of $\left(\phi^{n}(a)\right)$ such that for all $\varepsilon>0$ there is $k_{0}$ such that $\left\|\phi^{n_{k}}\right\| \leq \varepsilon$ when $k \geq k_{0}$. But then $n>n_{k}$ for $k \geq k_{0}$ implies

$$
\left\|\phi^{n}(a)\right\|=\| \phi^{n-n_{k}}\left(\phi^{n_{k}}(a)\|\leq\| \phi^{n_{k}} \|<\varepsilon .\right.
$$

Thus $\left\|\phi^{n}(a)\right\| \rightarrow 0$.

Conversely, if $\left\|\phi^{n}(a)\right\| \rightarrow 0$ then for $b \in C_{\phi}, \rho \in \mathscr{F}$

$$
\rho(a \circ b)=\rho\left(\phi^{n}(a \circ b)\right)=\rho\left(\phi^{n}(a) \circ \phi^{n}(b)\right) \rightarrow 0,
$$

for $n \rightarrow \infty$. Thus $\rho(a \circ b)=0$, completing the proof of the theorem.

It was shown in [5] that if $A$ is a $C^{*}$-algebra, and $P: A \rightarrow A$ is a faithful positive unital projection then the image $P(A)$ is a Jordan subalgebra of $A$. The following corollary proves more.

Corollary 3.3. Let $A$ be a $C^{*}$-algebra and $P: A \rightarrow$ A a faithful positive unital projection. Then $E_{P}=C_{P}=P(A)$. Hence $P(A)$ is in particular a Jordan subalgebra of $A$.

Proof. Since $P^{2}=P$ the orbit of each $a \in A$ is finite, so compact. Since $P$ is faithful the set of states $\mathscr{F}=\left\{\left.\omega\right|_{P(A)} \circ P\right\}$ with $\omega$ a state on $A$, is a faithful family of $P$-invariant states. Thus by Theorem 3.2 we have $E_{P}=C_{P}$. Since $P$ is a projection the only nonzero eigenvalue of $P$ is 1 , and the corresponding eigenoperators are the elements in $P(A)$. Thus $E_{P}=P(A)$, proving the corollary.

\section{REFERENCES}

1. Arveson, W., Asymptotic stability I: completely positive maps, Internat. J. Math. 15(3) (2004), 289-312.

2. Broise, B. M., letter to the author (1967).

3. Choi, M.-D., Positive linear maps on $C^{*}$-algebras, Thesis, University of Toronto (1972).

4. Choi, M.-D., Positive linear maps of $C^{*}$-algebras, Canad. J. Math. 24 (1972), 520-529.

5. Effros, E., and Størmer, E., Positive projections and Jordan structure in operator algebras, Math. Scand. 45 (1979), 127-138.

6. Evans, D., and Høegh-Krohn, R., Spectral properties of positive maps on $C^{*}$-algebras, J. London Math. Soc. 17 (1978), 345-355. 
7. Haagerup, U., and Størmer, E., Positive projections of von Neumann algebras onto JWalgebras, Rep. Math. Phys. 36 (1995), 317-330.

8. Kadison, R. V., A general Schwarz inequality and algebraic invariants for operator algebras, Ann. of Math. 56 (1952), 494-503.

9. Kadison, R. V., The trace in finite von Neumann algebras, Proc. Amer. Math. Soc. 12 (1961), 973-977.

10. Størmer, E., Positive linear maps of operator algebras, Acta Math. 110 (1963), 233-278.

DEPARTMENT OF MATHEMATICS

UNIVERSITY OF OSLO

0316 OSLO

NORWAY

E-mail: erlings@math.uio.no 